\title{
Hilfe für Patienten mit gemischten Schmerzformen
}

\begin{abstract}
— Tapentadol (PALEXIA ${ }^{\oplus}$ retard) vereint als erstes Analgetikum den Wirkmechanismus eines $\mu$-OpioidRezeptor-Agonisten (MOR) und eines Noradrenalin-Wiederaufnahme-Hemmers (NRI) in einem Molekül. Dabei geben Studien den „klaren Hinweis, dass es sowohl bei nozizeptiven als auch bei neuropathischen Schmerzen wirkt", sagte Prof. Ralf Baron, Leiter der Sektion Neurologische Schmerzforschung und -therapie der Klinik für Neurologie des Universitätsklinikums Kiel. Primär nozizeptiv bedingte Schmerzen aufgrund einer Kniegelenksarthrose gingen in einer offenen Phase-IIlbStudie um ca. 50\% zurück ( $p<0,0001$ [Steigerwald I et al. J Pain Res 2012;5:121-138]). Die Häufigkeit aller Nebenwirkungen war gering.
\end{abstract}

Rückenschmerzen deutlich gelindert Ein vergleichbarer Rückgang der Schmerzintensität zeigte sich in einer Phase-IIllb-Stu-

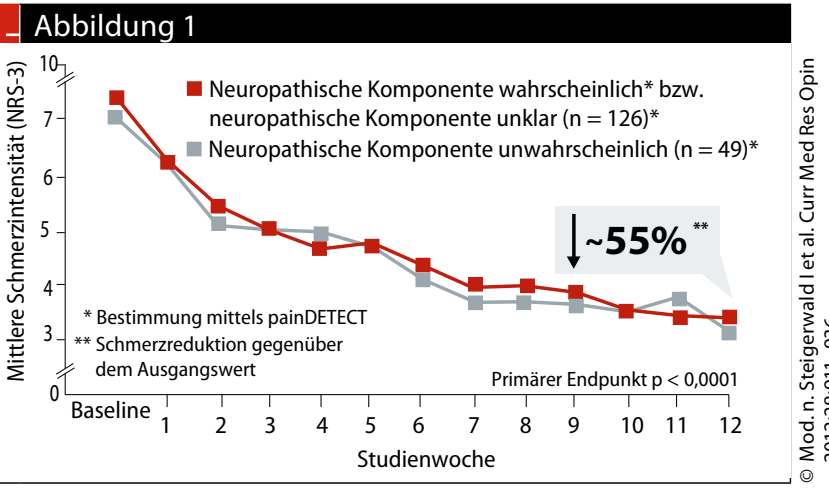

Tapentadol wirkt auch gegen die (wahrscheinlich) neuropathische Komponente chronischer Rückenschmerzen.

Zugelassenen Dosisbereich ausschöpfen

Wird ein opioidnaiver Patient auf Tapentadol eingestellt, sollte die Therapie mit $2 \times 50$ $\mathrm{mg} /$ Tag begonnen und alle drei Tage um $2 \times 50 \mathrm{mg}$ bis zur wirksamen Dosis auftitriert werden. Die zugelassene Höchstdosis von Tapentadol beträgt 2 × $250 \mathrm{mg}$. "Motivieren Sie den Patienten dazu, die Dosis weiter zu steigern", riet Dr. Kai-Uwe Kern, niedergelassener Schmerztherapeut

die mit Patienten mit chronischen lumbalen Rückenschmerzen - und damit einem der besten Beispiele für ein "Mixed Pain“-Syndrom, so Baron. Bei Patienten mit möglicher oder wahrscheinlicher neuropathischer Komponente besserten sich entsprechende Symptome wie brennende oder einschießende Schmerzen unter der Therapie erheblich [Steigerwald I et al. Curr Med Res Opin 2012;28:911-936] (Abb. 1). aus Wiesbaden. Bei vielen seiner Patienten trete im mittleren Dosisbereich noch eine weitere Besserung ein. Gerade bei neuropathischen Schmerzen seien Dosierungen von 300-350 mg Tapentadol täglich oft sehr gut wirksam.

Dr. Matthias Herrmann .

- Quelle: Symposium „Herausforderung in der Praxis: Chronische Schmerzen effektiv behandeln", Deutscher Schmerz- und Palliativtag, Frankfurt/M., März 2013 (Veranstalter: Grünenthal)

\section{Kurz notiert}

Protektion nach Ereignis - Die Grundlagen der Thrombozytenaggregationshemmung nach einem akuten Koronarsyndrom haben sich laut Priv.Doz. Holger Diedrichs, Frechen, durch die TRITON- und die PLATON-Studie grundlegend geändert. Die beiden Prüfsubstanzen, Prasugrel (Efient ${ }^{\oplus}$ ) und Tigagrelor, haben in den aktuellen Leitlinien der europäischen kardiologischen Gesellschaft (ESC) daraufhin eine IB-Empfehlung nach STEMI- und NSTEMI-Infarkt erhalten und werden damit noch vor Clopidogrel empfohlen. Für die Kombination mit Azetylsalizylsäure nach $\mathrm{PCl}$ erfolgte sogar eine IA-Empfehlung. Wichtig ist: Prasugrel scheint bei Diabetikern das Ereignisrisiko deutlich zu senken, was bei Tigagrelor nicht der Fall ist. Red. . - Nach Informationen von Daiichy Sankyo

\section{Akute Herzinsuffizienz}

\section{Konsequenzen werden oft unterschätzt}

—Etwa jeder fünfte Patient mit akuter Herzinsuffizienz (AHF) wird innerhalb von 30 Tagen erneut stationär aufgenommen und ebenso viele sterben im Verlauf von sechs Monaten. Wichtig sei es, die Patienten in der akuten Phase möglichst rasch zu rekompensieren, um die Organschäden zu begrenzen, sagte Prof. Stephan Felix, Direktor der Klinik für Innere Medizin am Universitätsklinikum Greifswald. Von großer Bedeutung sei dann aber auch die Erhaltungstherapie. Denn viele Patienten geraten nach der Klinikentlassung in eine Abwärtsspirale mit weiteren Exazerbationen nach Klinikentlassung.

Jetzt besteht die Hoffnung, mit RLX030 (Serelaxin), der rekombinanten Form des Schwangerschaftshormons Relaxin-2, eine Substanz gefunden zu haben, die die Prognose verbessern könnte. In der Studie RELAX-AHF (Efficacy and Safety of Relaxin for the Treatment of Acute Heart Failure) wurde bei insgesamt 1161 AHF-Patienten durch RLX030 die Dyspnoe der Patienten in den ersten fünf Tagen (primärer Endpunkt) signifikant stärker verringert als in der Placebogruppe [Teerlink et al., The Lancet 2013;381: 29-39]. Darüber hinaus ergab sich bei der Sicherheitsanalyse nach 180 Tagen ein signifikanter Mortalitätsvorteil für die Patienten der Serelaxingruppe mit einer Risikoreduktion um 37\%. Zudem wurde die Aufenthaltsdauer im Krankenhaus verkürzt (- 0,9 Tage), berichtete Prof. Karl Werdan, Internist am Universitätsklinikum Halle/Saale.

Roland Fath =

- Quelle: Symposium „Akute Herzinsuffizienz - ein unterschätztes Problem", Mannheim, April 2013, 79. Jahrestagung der Deutschen Gesellschaft für Kardiologie (Veranstalter: Novartis Pharma) 